\title{
Editor's Message to Special Issue of Young Researchers' Papers
}

\author{
RIN-ICHIRO TANIGUCHI ${ }^{1, a)}$
}

For the further advancement of information processing technologies, more support is needed for young researchers who are doing research driven by freely exploring ideas and/or by curiosity. One of the most important missions of the Information Processing Society of Japan (IPSJ) is to promote activities by young researchers, including students, who will lead the next generation. The Journal of Information Processing (JIP) has a series of special issues, starting in 2014, for promotion of young researchers. This special issue is the fifth one of the series, jointly with the IPSJ Journal, another journal of the IPSJ that receives manuscripts in Japanese.

The editorial committee of this special issue was organized by those who are experienced editorial board and committee members of JIP. They tried to help young researchers with publishing their research outcomes in a timely and speedy manner. The committee received 6 submissions until the deadline, June 16. In addition, the IPSJ Journal received another 34 submissions, so in total 40 manuscripts were submitted for the joint special issue. The first meeting was held online, and a meta reviewer was assigned to every submission. The second meeting was held on September 3, and it took nearly three months from the submission deadline to the notification of the first review result. The third meeting was held on November 22, and finally one article was accepted for publication, while the IPSJ Journal accepted 14 articles in Japanese. It took another three months from the first notification to the final one.

I would like to express my gratitude to the authors of the submitted manuscripts, the reviewers for careful and thorough reading, the editorial board and committee members for editing this special issue in a timely manner, and the IPSJ staff for supporting the special issue.

\section{The Editorial Committee}

\section{- Editor in-Chief:}

Rin-ichiro Taniguhci (Kyushu University)

- Associate Editor in-Chief:

Shin-ichi Minato (Kyoto University)
- Editorial Board Members:

Hiroyasu Matsushima (The University of Tokyo)

Masaki Shimaoka (SECOM Co., Ltd.)

Yuki Tanaka (Gunma University)

Hiroyoshi Watanabe (Teikyo University)

- Editorial Committee Members:

Masatsugu Ichino (The University of Electro-

Communications)

Masayuki Ihara (NTT Corp.)

Masataka Imura (Kwansei Gakuin University)

Daisuke Hatano (RIKEN)

Kaoru Katayama (Tokyo Metropolitan University)

Akihito Kitadai (J.F. Oberlin University)

Hiroyuki Manabe (Shibaura Institute of Technology)

Kiminori Matsuzaki (Kochi University of Technology)

Masanori Morise (Meiji University)

Hideharu Nakajima (NTT Corp.)

Masaaki Noro (Fujitsu Laboratories Ltd.)

Masao Ohira (Wakayama University)

Taizo Yamada (The University of Tokyo) 